\title{
SCHWINGER MODEL ON THE RANDOM BLOCK LATTICE
}

\author{
Ting-Wai CHIU ${ }^{1}$ \\ California Institute of Technology, Pasadena, CA 91125, USA
}

Received 25 July 1988; revised manuscript received 27 October 1988

\begin{abstract}
The fermion propagator and the vacuum polarization tensor of the Schwinger model are studied on the random block lattice, in the free field limit and weak coupling perturbation theory, respectively. The ensemble averaged fermion propagator and the vacuum polarization tensor agree with their respective values in the continuum theory for distances greater than three average lattice spacings. In momentum space, the ensemble averaged fermion propagator also agrees with the continuum propagator. These results support the possibility of having a chiral fermion interacting with the dynamical gauge field, on the random block lattice, with the correct continuum limit.
\end{abstract}

Recently, the properties of a free Dirac fermion have been studied on the four-dimensional random block lattice [1]. It has been shown that the random block lattice may provide a natural way to resolve the fermion doubling problem. The chiral symmetry of the doubled modes (at the tree level) is spontaneously broken due to the intrinsic short-range random coupling provided by the random block lattice. The fermions associated with the doubled modes acquire large masses of order of the inverse of the average lattice spacing $a$, and the approximate Goldstone boson arising from the broken symmetry has a mass of the order $\sqrt{m / a}$, where $m$ is the mass of the primary fermion. In the continuum limit they become infinitely heavy and therefore decouple from the theory. The effects of the doubled fermions extend only over a few lattice spacings. Similar results were also obtained earlier in 2D and 4D random lattices respectively [2].

It is natural to extend the study of the free fermion on the 4D random block lattice to incorporating the dynamical gauge fields. Then there are two different kinds of interaction on the lattice, namely the gauge interaction and the intrinsic random coupling. Since

\footnotetext{
This research is partially supported by US Department of Energy Grant No. DE-FG03-85ER25009, and by National Science Council, R.O.C.

I On leave of absence from Department of Physics, National Taiwan University, Taipei, Taiwan 10764, R.O.C.
}

the gauge interaction couples to the doubled modes as well as the primary fermion, it is not known to what extent the gauge interaction would modify the pattern of the chiral symmetry breaking of the doubled modes. What we really hope is that the gauge interaction does not alter the chiral symmetry breaking mechanism due to the intrinsic random coupling which only has realistic effects on the doubled modes. If that is the case, then we can regard the intrinsic random coupling as a background field which confines the doubled fermions. The only remaining problem is that the Goldstone boson arising from the chiral symmetry breaking of the doubled modes would couple to any pseudoscalar operator. Fortunately they can be suppressed by point-splitting the pseudoscalar operator. Before we plunge ourselves into any non-perturbative calculation of lattice gauge models including dynamical fermions, which is costly and time-consuming, it is instructive to study the interacting fermion in the weak gauge coupling limit, perhaps even on the 2D random block lattice. One of the most interesting models in the 2D continuum field theory is the quantum electrodynamics of a massless fermion, namely, the original Schwinger model [3], which is exactly soluble and exhibits the dynamical symmetry breaking phenomenon [4]. Perantonis and Wheater [5] have studied some interacting fermion models (without dynamical gauge fields) on a 2D random lattice. They found that the ultraviolet fine 
diagrams and the finite parts of the divergent diagrams have the correct continuum limits, while the ultraviolet divergent parts which receive contributions from the doubled modes diverge as the average lattice spacing goes to zero and thus create a fine tuning problem. Since the Schwinger model does not have any primitive divergent parts it is not plagued by these problems. Nevertheless I speculate that this fine tuning problem could be evaded in 4D models of fermions interacting through dynamical gauge fields, due to the inherent gauge symmetry. Recently Ren [6] has studied massless fermions on 2D random lattices with and without an external abelian gauge field respectively. The ensemble averaged fermion propagators agree well with the continuum limit.

In this paper, the properties of a massless fermion interacting with the abelian gauge field in the weak coupling perturbation theory on the $2 \mathrm{D}$ random block lattice are investigated. These results are compared to the continuum theory and thus serve as the first test whether we can have a chiral fermion interacting with the gauge field on the random block lattice. The most crucial quantity to study is the vacuum polarization tensor since the number of species of fermions must appear as a factor of it, and thus provides a good test for any scheme of the lattice fermion. In a recent study of the lattice Schwinger model, Bodwin and Kovacs [7] found that in the weak coupling perturbation theory all existing fermion schemes on the regular lattice have at least one shortcoming or more, when compared to the continuum theory. On the other hand, the results in this paper show that the Schwinger model on the random block lattice agrees with the continuum theory in the weak coupling perturbation theory.

On a 2D random block lattice, the action of the Schwinger model can be written as $\# 1$

$$
\begin{aligned}
A_{\mathrm{L}} & =\sum_{i} \sum_{\mu} \omega_{i} K_{i}^{\mu}\left[\bar{\psi}_{i} \gamma_{\mu} U_{\mu}(i) \psi_{i+\mu}\right. \\
& \left.-\bar{\psi}_{i} \gamma_{\mu} U_{\mu}^{+}(i-\mu) \psi_{i-\mu}\right] \\
& +\beta \sum_{p} w_{p}\left[1-\operatorname{Re}\left(U_{p}\right)\right]
\end{aligned}
$$

where $\psi_{i}$ and $\bar{\psi}_{i}$ are two independent 2-component spinors at site $i, \omega_{i}$ is the weight at site $i, K_{i}^{\mu}$ is the

\#1 A complete formulation of gauge models on the random block lattice will be given in a forthcoming paper [8]. inverse of the distance between the sites $i+\mu$ and $i-\mu$, $U_{\mu}(i)$ is the gauge link variable of the link $(i, i+\mu)$, and in the pure gauge action part, $w_{p}$ is the weight of the plaquette $\mathrm{P}, U_{p}$ is the ordered product of the link variables around the plaquette $P$ and $\beta=2 / g^{2}$. In terms of the coordinates of neighboring sites, they can be written as

$\omega_{i}=\frac{1}{4}\left(x_{i_{1}+1}^{1}-x_{i_{1}-1}^{1}\right)\left(x_{i_{2}+1}^{2}-x_{i_{2}-1}^{2}\right)$,

$K_{i}^{\mu}=\left(x_{i+\mu}-x_{i-\mu}\right)^{-1}$,

$U_{\mu}(i)=\exp \left[\mathrm{ig} l_{i}^{\mu} A_{\mu}\left(x_{i}+\frac{1}{2} l_{i}^{\mu}\right)\right]$,

$l_{i}^{\mu}=x_{i+\mu}-x_{i}$.

The sites of a 2D random block lattice are the cartesian product of two sets of randomized coordinates from each dimension. An example of a 2D random block lattice is shown in fig. 1 of ref. [1]. It is evident that there is no topological relationship between the random block lattice and the random lattice. The fundamental cell in the former is an $n$-rectangle but an $n$-triangle in the latter, where $n$ is the dimension. In the regular lattice limit the random block lattice goes to the hypercubical lattice but the random lattice goes to the hyper-equilateral-triangle lattice. The number of neighboring sites in the random lattice is much greater than the number $2 n$ in a random block lattice. This transcribes to the fact that the random coupling in the random lattice is stronger than that in the random block lattice. Nevertheless the random coupling in the random block lattice is already strong enough to break the chiral symmetry of the double modes and confine the effects of the doubled modes to the range of the order of average lattice spacing, and thus gives the correct continuum limit to the lattice action. This picture can be seen more clearly by mapping the action ( 1 ) into a $2 \mathrm{D}$ regular lattice action with random couplings. In the limit $\beta \rightarrow \infty$ the gauge links are frozen, then the positive random coupling between neighboring spinors is $\omega_{i} K_{i}^{\mu}$ which obeys the Poisson distribution with a mean value equal to one-half of the average lattice spacing. In the regular lattice limit, the random coupling becomes a constant and thus this short-range strong interaction vanishes and then the chiral symmetry of the doubled modes is restored. Similar arguments should be applicable to models on the random lattice. However, field theoretic calculations on the random block 
lattice are much simpler than their counterparts on the random lattice. Furthermore the hyperrectangular structure of the random block lattice enables all models formulated on it to have high efficiency in parallel computations which must be the trend of future generations of supercomputers.

In the Schwinger model, the fermion spinor field is confined and all its excitations are absent from the physical spectrum. The only remnant of this fundamental fermion is the existence of a free neutral scalar with mass $g / \sqrt{\pi}$, which is the fermion-antifermion bound state. Therefore we can only study the fermion propagator in the limit $\beta \rightarrow \infty$ where all gauge link variables are frozen, that is the free fermion action

$$
\begin{aligned}
& A_{\mathrm{L}}^{(0)}=\sum_{i} \sum_{\mu} \omega_{i} K_{i}^{\mu} \bar{\psi}_{i} \gamma_{\mu}\left(\psi_{i+\mu}-\psi_{i-\mu}\right) \equiv \bar{\Psi} \gamma_{\mu} T_{\mu} \Psi, \\
& \left(S_{\mathrm{F}}^{\mathrm{L}}\right)_{i j}=\left\langle\psi_{i} \bar{\psi}_{j}\right\rangle=\left(\gamma_{\mu} T_{\mu}\right)_{i j}^{-1}=\gamma_{\mu}\left(T_{\mu}\right)_{i k}\left(T^{2}\right)_{k j}^{-1} \\
& \quad \equiv \gamma_{\mu}\left(S_{\mu l}^{\mathrm{L}}\right)_{i j} .
\end{aligned}
$$

The fermion propagator is then the inverse of $\gamma_{\mu} T_{\mu}$ which corresponds to the fermionic momentum operator on the lattice. On a given lattice, the propagator $\left(S_{\mathrm{F}}^{\mathrm{L}}\right)_{i j}$ is proportional to the probability amplitude of the transition from site $i$ to site $j$ on this particular lattice. In other words, it is the sum of the probability amplitude over all possible paths between the site $i$ and the site $j$. In the spacetime continuum, many other paths are possible. In order to take into account other paths which are allowed in the continuum, we must generate many different random block lattices while holding the sites $i$ and $j$ fixed, and calculate the propagator in each of these lattices. Since each random block lattice is equally probable, the resulting propagator is then the arithmetic average of the propagators over an ensemble of random block lattices. This ensemble averaged propagator would agree with the continuum propagator of the same boundary conditions, provided that the inclusion of the unconsidered paths does not change the ensemble average significantly. In practice, we only perform the ensemble average over the random block lattices having the same number of sites in each dimension respectively.

For symmetric configurations (the endpoints of the propagator lying on the diagonal $\left.x_{1}=x_{2}\right), S_{1}^{\mathrm{c}}(x)=$ $S_{2}^{\mathrm{c}}(x)$, where the superscript c denotes continuum.
Then the ensemble average $\left\langle S_{1}^{L}(x)\right\rangle$ would be almost equal to $\left\langle S_{2}^{\mathrm{L}}(x)\right\rangle$. The results of the ensemble average $\left\langle S_{\mu}^{\mathbf{L}}(x)\right\rangle$ over 1000 random-block lattices, each of $40 \times 40$ sites and with the endpoints of the propagator fixed, are plotted in fig. 1 . Since there is almost no difference between $\left\langle S_{1}^{\mathrm{L}}(x)\right\rangle$ and $\left\langle S_{2}^{\mathrm{L}}(x)\right\rangle$ for all values of $x$, we do not distinguish them by using different symbols in fig. 1. For distances greater than two average lattice spacings, they agree with the continuum propagator excellently. For the asymmetric configurations (the endpoints of the propagator lying on the line $x_{1}=2 x_{2}$ ), the ensemble averaged propagator agrees with the continuum propagator for distances greater than three average lattice spacings.

It is also instructive to study the fermion propagator in the momentum space. For a given momentum $p=\left(p_{1}, p_{2}\right)$, the Fourier transformation is evaluated on a random block lattice to be as follows:

$$
\tilde{S}_{\mu}(p)=\sum_{i} \omega_{i} \exp \left(\mathrm{i} p \cdot x_{i}\right) S_{\mu}\left(x_{i}\right)
$$

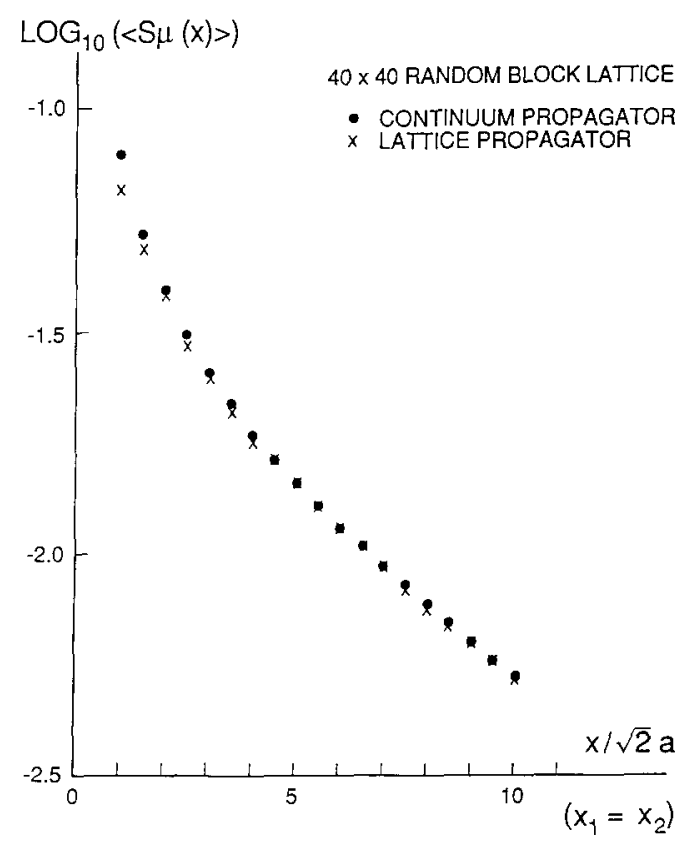

Fig. 1. In the limit $\beta \rightarrow \infty$, the ensemble average of the massless fermion propagator over $10002 \mathrm{D}$ random block lattices, each of $40 \times 40$ sites and with periodic boundary conditions. The crosses are the ensemble averaged propagator while the dots are the continuum propagator with the same boundary conditions. The endpoints of the propagator are lying on the diagonal $x_{1}=x_{2}$, 
where $S_{\mu}(x)$ is obtained according to (4). Then the ensemble average $\left\langle\tilde{S}_{\mu}(p)\right\rangle$ is the arithmetic average over an ensemble of random block lattices. For symmetric positive momenta $\left(p_{1}=p_{2}>0\right)$, the ensemble average over 1000 random block lattices, each of $40 \times 40$ sites and with periodic boundary conditions are plotted in fig. 2. Since there is almost no difference between $\left\langle\bar{S}_{1}(p)\right\rangle$ and $\left\langle\widetilde{S}_{2}(p)\right\rangle$ for all values of $p$, we do not distinguish them by using different symbols. Except at small momenta, the agreement between the ensemble average $\left\langle\tilde{S}_{\mu}(p)\right\rangle$ and the continuum propagator is excellent. The discrepancies at small momenta are essentially due to the fluctuations of the low energy modes. This can be understood by looking at the singularities caused by the zero modes in the matrix inversion of eq. (4). A small regulator mass $\left(10^{-10}\right)$ has been added to $T^{2}$ to avoid the numerical singularities. The ensemble averages are of course independent of the regulator mass provided it

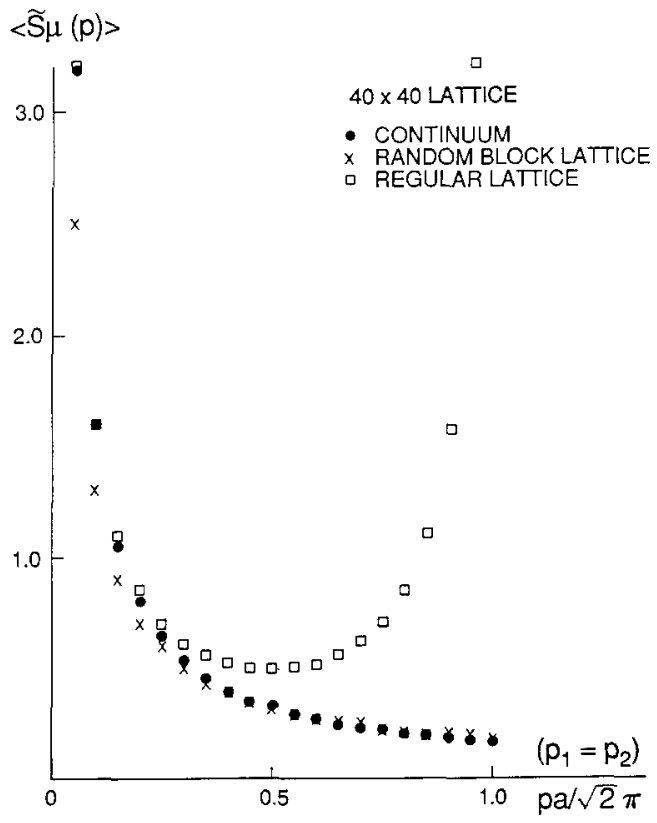

Fig. 2. Massless fermion propagator in momentum space, in the limit $\beta \rightarrow \infty$. The Fourier transform is calculated according to eq. (5), and the ensemble average is over $10002 \mathrm{D}$ random block lattices, each of $40 \times 40$ sites and with periodic boundary conditions. The ensemble averaged propagator is denoted by the crosses while the continuum propagator with the same boundary conditions is denoted by dots, and the regular lattice propagator by squares. is small enough. These discrepancies at small momenta can be diminished by increasing the number of random block lattices for ensemble averaging. (For the massive fermion propagator, the low energy modes do not cause any numerical instability and therefore the ensemble averaged propagator agrees with the continuum propagator excellently at small momenta.) For comparison, the massless fermion propagator on a regular lattice of the same size and boundary conditions is also plotted. At small momenta, the regular lattice propagator agrees with the continuum propagator excellently. But it starts to deviate at $p_{1}=p_{2}=0.3 \pi / a$, and rises to $\infty$ at the pole $p_{1}=p_{2}=\pi / a$, which is one of the well-known fermion doubled modes on the regular lattice (the rest of the doubled modes are at $(0, \pi / a)$ and $(\pi / a, 0)$, which are of course not shown in fig. 2). The effects of the Goldstone bosons arising from the spontaneous chiral symmetry breaking of the doubled modes are almost decoupled from any component of the massless fermion propagator, even at large momenta $(p \gtrsim 1 / a)$. This amazing fact is only true for the massless fermion propagator in momentum space because the leading order contribution of the doubled modes to the free fermion propagator in momentum space transforms like a scalar or a mass term and thus does not contribute to the vector-like components of a free massless fermion propagator in momentum space. If we examine the propagator of a free massive fermion, then we can see explicitly that the mass component

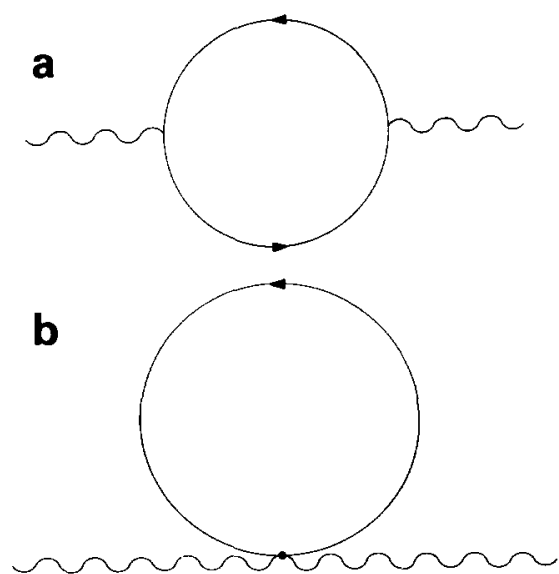

Fig. 3. The Feynman diagrams contributing to the lowest order vacuum polarization tensor in the lattice Schwinger model. Diagram (b) does not exist in the contiuum theory. 
receives contributions from these Goldstone bosons, especially at large momenta, while the vector-like components are in good agreement with the continuum propagator $p_{\mu} /\left(p^{2}+m^{2}\right)$.

In the weak gauge coupling limit, the action (1) can be expanded in $g$ to yield the Feynman rules on the random block lattice. The most important quantity in the Schwinger model is the vacuum polarization tensor which consists of two parts corresponding to figs. $3 a$ and $3 b$, respectively. In the position space, they are

$$
\begin{aligned}
\pi_{\mu \nu}^{(a)} & \left(x_{i}+\frac{1}{2} l_{i}^{\mu}, x_{j}+\frac{1}{2} l_{j}^{\nu}\right) \\
& =-\frac{1}{2} g^{2}\left\{\omega_{i} \omega_{j} K_{i}^{\mu} K_{j}^{\nu} l_{i}^{\mu} l_{j}^{\nu} \operatorname{tr}\left[\gamma_{\mu}\left(S_{\mathrm{F}}^{\mathrm{L}}\right)_{i+\mu, j} \gamma_{\nu}\left(S_{\mathrm{F}}^{\mathrm{L}}\right)_{j+\nu, i}\right]\right. \\
& +\omega_{i} \omega_{j+\nu} K_{i}^{\mu} K_{j+\nu}^{\nu} l_{i}^{\mu} l_{j}^{\nu} \operatorname{tr}\left[\gamma_{\mu}\left(S_{\mathrm{F}}^{\mathrm{L}}\right)_{i+\mu, j+\nu} \gamma_{\nu}\left(S_{\mathrm{F}}^{\mathrm{L}}\right)_{j, i}\right] \\
& +\omega_{i+\mu} \omega_{j} K_{i+\mu}^{\mu} K_{j}^{\nu} l_{i}^{\mu} l_{j}^{\nu} \\
& \times \operatorname{tr}\left[\gamma_{\mu}\left(S_{\mathrm{F}}^{\mathrm{L}}\right)_{i, j} \gamma_{\nu}\left(S_{\mathrm{F}}^{\mathrm{L}}\right)_{j+\nu, i+\mu}\right] \\
& +\omega_{i+\mu} \omega_{j+\nu} K_{i+\mu}^{\mu} K_{j+\nu}^{\nu} l_{i}^{\mu l} l_{j}^{\nu} \\
& \left.\times \operatorname{tr}\left[\gamma_{\mu}\left(S_{\mathrm{F}}^{\mathrm{L}}\right)_{i, j+\nu} \gamma_{\nu}\left(S_{\mathrm{F}}^{\mathrm{L}}\right)_{j, i+\mu}\right]+(\mu \leftrightarrow \nu)\right\},
\end{aligned}
$$

$$
\begin{aligned}
& \pi_{\mu \nu}^{(\mathrm{b})}\left(x_{i}+\frac{1}{2} l_{i}^{\mu}, x_{j}+\frac{1}{2} l_{i}^{\nu}\right) \\
& \quad=g^{2} \delta_{i j} \delta_{\mu \nu} \omega_{i} K_{i}^{\mu}\left\{\left(l_{i}^{\mu}\right)^{2} \operatorname{tr}\left[\gamma_{\mu}\left(S_{\mathrm{F}}^{\mathrm{L}}\right)_{i+\mu, i}\right]\right. \\
& \left.-\left(l_{i-\mu}^{\mu}\right)^{2} \operatorname{tr}\left[\gamma_{\mu}\left(S_{\mathrm{F}}^{\mathrm{L}}\right)_{i-\mu, i}\right]\right\} .
\end{aligned}
$$

For asymmetric configurations (endpoints lying on the line $\left.x_{1}=2 x_{2}\right), \pi_{11}^{\mathrm{c}}=-\pi_{22}^{\mathrm{c}}$. In fig. 4, the ensemble averages $\left(\left|\left\langle\pi_{11}\right\rangle\left\langle\pi_{22}\right\rangle\right|\right)^{1 / 2}$ and $\left\langle\pi_{12}\right\rangle$ over a 1000 $40 \times 40$ random block lattices and the corresponding values in continuum theory are plotted versus the distances. The gauge coupling factor $g^{2}$ as well as the normalization factor of each term in eq. (6) have been factored out before doing the ensemble averaging. For point splitting equal to zero, the ensemble averages are almost four times the corresponding values in the continuum theory. The difference is due to the presence of the three species of Goldstone pseudoscalars, arising from the chiral symmetry breaking of the doubled modes. The effects of these pseudoscalars can be suppressed by splitting the endpoints. For example, the endpoints $\left(x_{1}, x_{2}\right)$ and $\left(y_{1}, y_{2}\right)$ of the forward moving propagator, are split from the
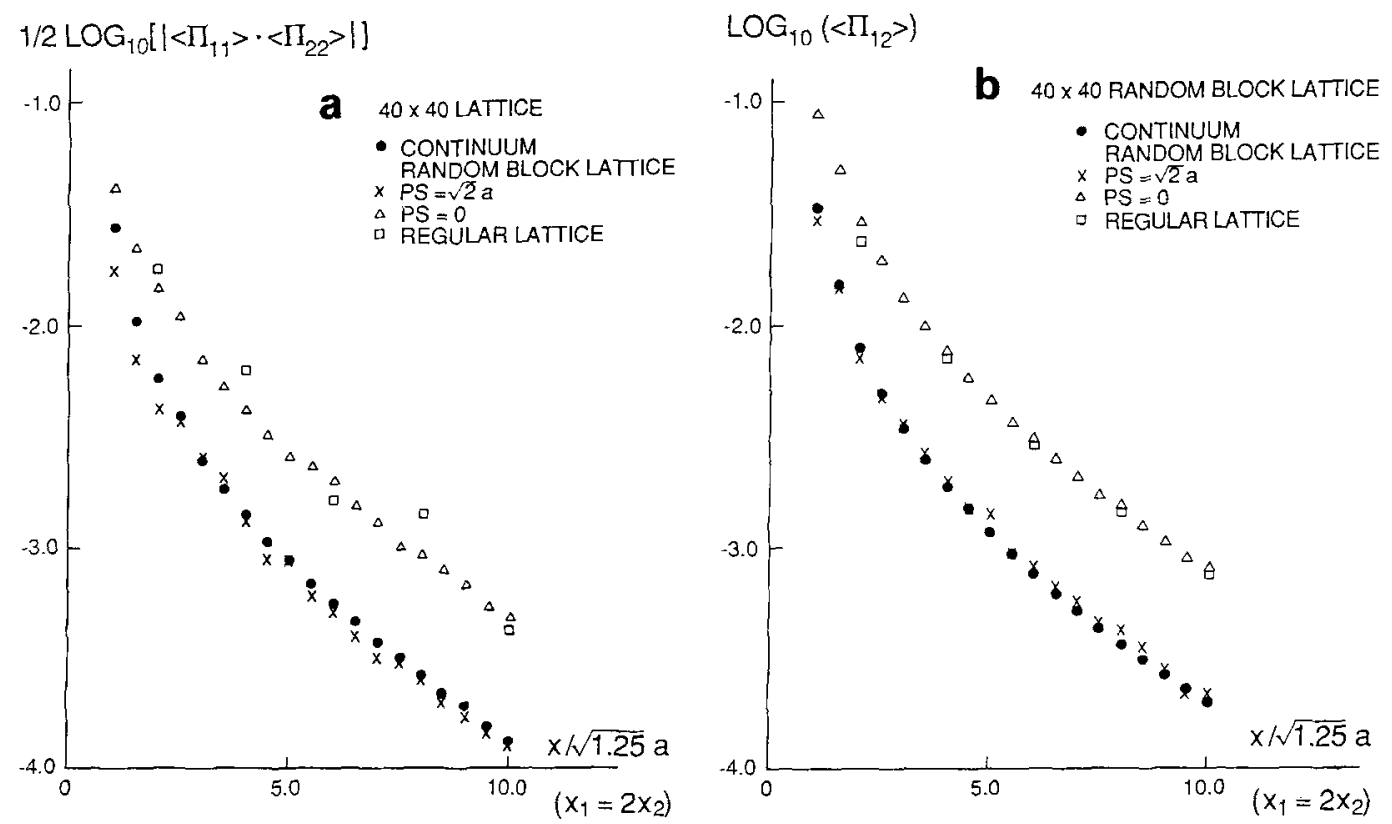

Fig. 4. Vacuum polarization tensors with endpoints lying on the line $x_{1}=2 x_{2}$. The ensemble average is over $10002 \mathrm{D}$ random block lattices, each of $40 \times 40$ sites and with periodic boundary conditions. The ensemble averaged vacuum polarization tensor with pointsplitting ps $=\sqrt{2} a$ agrees with the continuum theory, while the one without point-splitting is about four times the one of the continuum theory, the same as the vacuum polarization tensor on a regular lattice with the same size and boundary conditions. (a) $\left(\mid\left\langle\pi_{11}\right\rangle \times\right.$ $\left.\left\langle\pi_{22}\right\rangle \mid\right)^{1 / 2}$. (b) $\left\langle\pi_{12}\right\rangle$. 
endpoints $\left(x_{1}-a, x_{2}+a\right)$ and $\left(y_{1}-a, y_{2}+a\right)$ of the backward moving propagator. In fig. 4 , such a pointsplit vacuum polarization tensor on the random block lattice agrees with the continuum limit very well for distances greater than three average lattice spacings. The agreement implies that the gauge boson in the random block lattice also acquires a mass $g / \sqrt{\pi}$ through the same dynamical symmetry breaking mechanism as the continuum theory, in spite of the presence of the doubled modes.

To summarize, numerical results of the ensemble averages of the massless free fermion propagator and the vacuum polarization tensor in the weak gauge coupling limit provide substantial evidence that on the random block lattice, a chiral fermion coupled minimally to the gauge field, could have the correct continuum limit. Since the randomness in a random lattice plays the same role as the randomness in a random block lattice, it is intuitive for one to infer that the results presented in this paper must hold for the same model on the random lattice as well as any reasonable variant of the random lattice.
It is a pleasure to thank Professor Geoffrey C. Fox for his support and kind hospitality.

\section{References}

[1] T.W. Chiu, Phys. Lett. B 206 (1988) 510.

[2] D. Espriu, M. Gross, P. Rakow and J, Wheater, Nucl. Phys. B 275 ( 1986 ) 39; Prog. Theor. Phys. Suppl. 86 (1986) 04; R. Friedberg, T.D. Lee and H.C. Ren, Prog. Theor. Phys. Suppl. 86 ( 1986) 322;

Y. Pang and H.C. Ren, Phys. Lett. B 172 (1986) 392; B 195 (1987) 223.

[3] J. Schwinger, Phys. Rev. 128 (1962) 2425.

[4] J. Lowenstein and J. Swieca, Ann. Phys. (NY) 68 (1971) 72.

[5] S.J. Perantonis and J.F. Wheater, Nucl. Phys. B 295 (1988) 443.

[6] H.C. Ren, Massless fermions on random lattices, Rockefeller University preprint DOE/ER/40325-28 (1988).

[7] G.T. Bodwins and E.V. Kovacs, Phys. Rev, D 35 (1987) 3198.

[8] T.W. Chiu, Gauge theories on the random block lattice, forthcoming paper. 